\section{THE DIAGNOSIS OF THE COMMONER CAUSES} OF CHRONIC JAUNDICE.*

By P. J. CAMMIDGE, M.D.Lond.

SINCE jaundice is due to obstruction of the free flow of bile into the intestine, it is only a symptom, and, if a successfal line of treatment is to be followed, it is necessary that the nature and situation of the obstruction should be diagnosed as speedily and accurately as possible.

Two principal types of jaundice are met with: (1) Those in which there is gross and frank blocking of the larger bile ducts, without abnormal blood destruction; (2) those in which the obstruction is due to an increase in the viscidity of the bile in the smaller ducts, and in which there is a pathological destruction of red blood cells. In the first group the obstruction may be due to: (a) Me. chanicel blocking of the lamen of the ducts by gall stones, malignant growths, or stricture, or by an infiammatory swelling of the mucous membrane or lymphoid tissue at the duodenal outlet; (b) to compression of the ducts from without by swellings of the pancreas, enlarged lymph ylands in the portal fissure, lesions of the stomach, duocenum, kidney, etc., or by pressure from an aneurysm of the aorta, hepatic or mesenteric arteries, and other rarer causes, such as uterine tumours, etc. In the second, or toxaemic, group the jaundice occurs as an incidental com. plication in the course of an infectious disease such as pyremia, septicaemia, relapsing fever, pneumonia, scarlet fever, epidemic jaundice, Weil's disease, acute yellow atrophy, or as the result of the action of certain poisons or drugs such as phosphorus, toluylendiamene; snake poison, chloroform, chloral, acetanilide, santonin, arseniuretted hydrogen, aniline, copper sulphate, mushrooms, etc.

More than four-fifths of all cases of janndice met with in ordinary practice belong to the first group, the obstruction to the bile flow being of an obvious mechanical character, the most common causes, in my experience, being biliary calcali and malignant disease of the pancreas. On classifying 400 cases of jaundice that I have had the opportunity of investigating during the past five years, I find that in 46 per cent. it was due to the former cause, and in 28 per cent. to the latter, so that in nearly 70 per cent. of my cases the diagnosis lay between gall stones and malignant disease of the pancreas. Catarrhal janndice was diagnosed in 15 per cent. of my cases, a growth of the bile ducts or gall bladder in 3 per cent.; cirrhosis of the head of the pancreas, without gall stones, in 4 per cent.; cirrhosis of the liver in 4 per cent., secondary growths in the liver 3 per cent., and in 2 per cent. the jaundice depended upon rarer causes, such as stricture of the duct, etc. In 93.5 per cent. of the gall-stone cases the calculi were found in the common bile duct at operation, and in 6.5 per cent. they were only present in the gall bladder or cystic duct, the jaundice in the latter being apparently due to an associated cholangitis. I may remind you in passing that gall stones may be present in the common bile duct without producing jaundice, and that unless this is borne in mind, the symptoms to which they give rise may be attributed to the wrong cause, notably to "indigestion." In 32 per cent. of my cases of common duct cholelithiasis there was no jaundice, and in several of these the symptoms had culminated in glycosuria, owing to the long continued irritation of the pancreas, which had been allowed to progress unrecognized and unrelieved.

In many instances the history of the case is suggestive of the cense of the obstruction. A sense of weight or distension in the upper part of the abdomen, generally coming on a short time after meals, which is relieved by eructation or vomiting, and is often noticed to be particularly liable to succeed indulgence in certain articles of diet, a gnawing feeling below the ensiform cartilage, a catch in the breath or an acute stabbing pain under the right costal margin on deep inspiration, a feeling of chilliness, or even a slight rigor, are among the prodromal symptoms of cholelithiasis. It will be noted how easily these may be attributed to "indigestion." In such cases the jaundice will have generally appeared suddenly. It may have been preceded or accompanied by paroxysmal

* A paper read before the Hampstead Division of the British Medica Association, February 10th, 1911. attacks of pain starting in the right hypochondriam or in the epigastrium, radiating thence over the abdomen and through to the right scapular region, but in practice typical gall-stone colic is a comparatively rare symptom. If the intensity of the jandice fluctuates, deepening after each paroxysm of pain and diminishing in the intervals, if there is fever and there are ague-like attacke, gall-stone obstruction is highly probable. Jaundice coming on painlessly, insidiously, and persistently, without intermission, in a previously healthy individual, with no history of abdominal or digestive symptoms, persistent loss of flesh, and marked failure of strength, are suggestive of growth. The character of the jaundice is often indicative of the nature of the obstruction. In all forms of icterus the bile pigment in the tissues is at first in solution, but after a considerable amount has accumulated it may be deposited in a granular form. The deposits of bilirubin then undergo slow oxidation, the colour changing from yellow to an olive or dirty greyish-green, hence the skin appears of a dark green or slate colour, the so-called "black jaundice." Since the obstruction of the bile ducts is more complete and persistent in malignant disease of the pancreas than when it is due to gall stones, "black" jaundice is suggestive of the blocking of the common bile duct by a growth.

Examination of the abdomen will frequently reveal tenderness and rigidity of the right rectus muscle after an attack of gall-stone colic, but distension of the gall bladder is usually absent. A distended gall bladder with jaundice is usually dependent upon malignant disease of the head of the pancreas or common bile duct: Dilatation of the gall bladder may, however, be occasionally due to impacted gall stones in the common duct, but in such cases the distension is as a rule of short duration, since the inflam. matory softening of the walls of the duct ultimately allows the passage of the retained mucus and bile, whereas in malignant disease the gall bladder steadily enlarges as the jaundice intensifies. A stone impacted in the cystic duct, stricture of the duct, acute inflammation of the gall bladder, or chronic inflammation of the head of the pancreas, when there is also a stone in the common duct, or a stone in the cystic duct pressing on the common duct, may give rise to a distended gall bladder with jaundice. On the other hand, when the gall bladder has been more or less completely obliterated by previous attacks of inflammation or is congenitally absent, distension cannot occur when the common bile duct is obstructed by a malignant growth. Enlargement of the liver as the result of the biliary stasis is frequently to be made out. In gall-stone cases the liver has a smooth surface and varies in size and tenderness with the intensity of the jaundice. In obstruction due to growth it is often enlarged and may be tender, but the size does not vary. If the surface is irregular and nodular and there is also free fluid in the abdomen and oedema of the feet, advanced cancer of the head of the pancreas is indicated. The presence of small angiomata in the skin, particularly of the abdomen, is a point in favour of cancer. An oblong swelling, due to the enlarged pancreas, can sometimes be felt on deep pressure in the lower epigastric region, especially when the patient is under an anaesthetic. This may be due to malignent disease of the gland, but is more commonly an inflammatory swelling. If the former, it is hard, irregular, and painless; if the latter, it is often not so hard, is smooth, and is generally painfal. Chronic pancreatitis is frequently accompanied by attacks of pain resembling those due to gall stones, but the pain in such cases radiates to the left side and is often more severe under the left shoulder blade or in the mid.scapular region.

In cases where there is a characteristic history, and the signs and symptoms are typical, a satisfactory diagnosis may be made from the clinical conditions alone, partica. larly when the presence of gall stones is indicated, but a diagnosis of malignant disease must always be made with caution, for there are not a few cases in which the clinical evidence points to carcinoma of the pancreas, in which the jaundice is in reality due to an inflammatory swelling of the head of the gland, with or without an associated gallstone obstruction. It is practically impossible to distinguish by clinical methods alone between cancer of the head of the pancreas and growths of the common bile duct or ampulla of Vater. But in many cases of chronic jaundice 
the history is either unreliable or not characteristic and the symptoms are conflicting or confusing. In such doubtful and difficult cases, great assistance in arriving at a reliable opinion may be obtained by a thorough investigation of the digestive and metabolic distarbances revealed by an analysis of the urine and faeces.

Before considering the results and indications yielded by such investigations, it is advisable that we should briefly consider the anatomy and pathology of the common bile duct and its related structures, for unless these are thoroughly appreciated the significance of the chemical changes met with in jaundice due to various causes is not always clear. Commencing at the junction of the cystic and hepatic ducts, the common bile duct courses along the free border of the lesser omentum with the portal vein and hepatic artery; it then passes behind the first part of the duodenum, the portal vein lying to its inner side and the inferior vena cava behind. These sections of the duct are known as the supraduodenal and the retroduodenal portions respectively. The duct now comes into relation with the pancreas, to which it is closely applied, being completely surrounded by pancreatic tissue in about 68 per cent. of cases, and lying in a groove in the posterior surface of the gland in the remaining 32 per cent. The anatomical relations of this, the pancreatic portion of the common duct, are of considerable importance since pathological conditions within the duct are liable to involve the pan. creas, and conversely swellings of the head of the pancreas may cause obstruction of the duct, particularly in those instances where the duct passes through the substance of the gland. In its fourth, intraparietal, or interstitial portion the common bile duct enters the wall of the second part of the duodenum, the muscular layers of which it traverses. Beneath the mucous membrane it dilates into a small reservoir known as the ampulla of Vater. The main pancreatic duct, or duct of Wirsung, usually opens into the ampalla of Vater distal to the common bile duct but separated from it by a small transverse fold of mucous membrane. The ampulia communicates with the duodenum by a small round or elliptical orifice, situated about 3 or 4 inches from the pylorus. This apertare is the narrowest part of the bile duct, and it is here that gall stones which have successfully negotiated the common bile duct are apt to lodge and cause the sudden onset of jaundice which is so characteristic. If the stone is large enough to fill the ampalla of Vater it will also ocolude the pancreatic duct, preventing the entry of the pancreatic ferments into the intestine and bringing about a catarrhal pancreatitis from the back pressure, and subsequent infection of the retained secretions. The average length of the ampulla is about one-sixth of an inch, and it is nsually about one-fifth to one-sixth of an inch in breadth, bat it is liable to considerable variation, occasionally being nearly half an inch long. A small stone impacted in the duodenal orifice may not entirely fill the cavity of an unusually large ampulla, and the bile and pancreatic ducts may thus be converted into a continuous channel. Such a condition has been described by Opie, and he has demonstrated that the passage of bile into the pancreatic duct, which is possible in such a case, may bring about acute inflammation of the pancreas Subsequently Flexner showed that if the bile is modified by a diminution of its salts, or an increase in its colloidal elements, such as occurs in chronic inflammatory conditions of the bile passages, chronic pancreatitis results.

The accessory pancreatic duct, or duct of Santorini, opens in the duodenum about three'quarters of an inch above the bile papilla. Since the accessory duct anastomoses with the main duct within the pancreas in about 90 per cent. of cases, it has been assumed that it may act as a safety valve, and prevent the effects of back-pressure when the duct of Wirsung is obstructed, but as the duodenal orifice of the accessory duct is obliterated, or markedly constricted in about 50 per cent. of cases, it is probable that in at least that proportion it cannot assume the functions of the main duct; moreover, the duct of Santorini may itself be compressed by moderate-sized gall stones in their passage through the pancreatic portion of the common bile duct.

The mechanical view, and even the chemical explanation of Opie and Flexner, of the relation between gall stones and pancreatitis only partly accounts for the pathology of these conditions in my opinion. In both the action of micro-organisms on the damaged tissues is not taken into account. In some instances $I$ believe that the affections are primarily microbic, the disease of the bile channels and pancreas arising from invasion of these structures by intestinal organisms, which in the case of the pancreas activate the pancreatic secretion and set up autolytic changes. Normally the entry of microorganisms into the duct of Wirsung is prevented by the flow of the secretion and the presence of valve-like folds in the walls of the diverticulum of Vater, but if the passage of bile and pancreatic juice is interfered with, and the valves are obliterated or damaged, organisms may find their way directly from the duodenum into the ducts. If from any cause, such as an intestinal catarrh, the virulence and number of the intestinal bacteria are increased an inflammatory condition of the bile and pancreatic dacts is apt to follow, originating in the one case cholangitis and gall stone formation and in the other chronic pancreatitis.

In the 50 per cent. or so of cases where the accessory pancreatic duct is patent a more open route for the infection of the pancreatic ducts, and through them of the bile ducts, exists, for there are no valves at the entrance to the duct of Santorini, and, according to Desjardins, the secretion may flow indifferently from the panoreas to the intestine or from the intestine to the gland. Organisms carried in from the duodenum by a reverse current will be conveyed through the head of the pancreas to the point where the ducts of Santorini and Wirsung anastomose, and there, meeting with the direct ourrent from the main duct, be carried into the ampulla of Vater, to pass into the intestine again, or along the common bile duct, according to the conditions that are present. The effects of an arrest of septic organisms will first be evident in the bile ducts, for these, unlike the pancreatic ducts, are not more or less immune from the constant presence of intestinal bacteria. When the virulence of these organisms has been increased by growing in the morbid secretions and diseased tissues of the bile ducts the immunity of the pancreas may be overcome and pancreatitis follow. Inocalation and reinoculation from the pancreas to the bile passages, and back from the bile passages to the pancreas, will then occur so that the pancreatic lesion will continually progress so long as the obstruction of the common duct is unrelieved. When the blocking of the ducts by gall stones is complete the inflamed walls present a ready path by which infection may travel from the intestine, and we consequently find cholangitis and an infected condition of the bile in practically every case. Absolute blocking of the common bile duct is, however, very uncommon, except in cancer of the head of the pancreas, for, as we shall see later, bile pigment can be found chemically in the faeces in nearly every case of gall stones, even when the stools appear white to the naked eje.

Beside the direct route of entry by way of the bile papilla and accessory pancreatic duct, intestinal organisms may reach the bile ducts through the portal vein. This is probably one of the ways in which simultaneous gall stone formation and damage of the pancreas are initiated, and acconnts for the inflammatory changes in the pancreas being more marked in some instances than might be expected from the length of time the mechanical obstruction of the ducts has been known to exist.

The examination of the urine from a case of jaundice should be as complete as possible, in order that every abnormality which it will show may be discovered and considered in arriving at an opinion. It is as misleading to rely on one test as it would be clinically to diagnose scarlet fever only by examining the tongue. The points to which I usually pay special attention are: The presence of bile, urobilin, sugar, albumen, an excess of indican, and the results of the "pancreatic" reaction.

Bile.

A very large number of tests for bile in the urine have been described, but after trying most of them I have come to the conclusion that Rosenbach's modification of Gmelin's nitric acid test is the simplest and most reliable. If a sufficiently large quantity of urine is filtered through the same filter paper the test is as delicate as many more complicated methods. It must be borne in mind that it is not always possible to detect bile pigment in the urine, even with the most delicate test, in the early stages of an attack of jaundice and later when the pigmentation of the 
skin is clearing up. In the former an examination of the blood serum may be helpful in arriving at a correct conclusion, but the simplest test is to examine the conjunctivae. If a distinct icteric tinge is discovered by daylightnot artificial light, by which the coloration is not so well seen-it shows that there is some obstruction to the free flow of bile into the intestine.

\section{Urobilin.}

The presence of urobilin in the urine, as shown by the alcoholic zinc acetate test, points to there being interference with the functions of the liver. If abnormal blood destruction can be excluded, a positive reaction is suggestive of cholangitis, which, if there is distinct jaundice, is probably due to the presence of gall stones in the common bile duct. If the jaundice is only slight, and there is also evidence of pancreatitis and intestinal catarrh, the cholangitis may be due to an ascending infection of the bile ducts from the duodenum. A reaction for urobilin is comparatively ancommon in malignant disease of the pancreas and bile ducts, and when present points to the existence of secondery growths in the liver.

\section{Indican.}

An excess of indican in the urine is suggestive of there being abnormal putrefactive changes in the contents of the upper part of the intestine, with catarrh of the walls. From the supposed antiseptic action of the bile it might be expected that in all cases where it is prevented from entering the intestine such abnormal putrefactive changes would occur, but an excess of indican is rarely met with in cancer of the head of the pancreas, where the blocking of the bile duct is generally absolute, and in cases where there are impacted gall stones in the common bile duct it is also uncommon. It is rather more frequent when the stones are "floating" in the common duct, but is most common with "catarrhal" jaundice where the obstraction is due to swelling of the mucous membrane and lymphatic tissue in and around the biliary papilla, or to swelling of the head of the pancreas, secondary to an ascending infection from the drodenum.

\section{Sugar.}

The presence of sugar in the urine of a case of chronic jaundice is almost pathognomonic of serious disease of the pancreas which may be malignant or inflammatory. I have met with glycosuria in 7.5 per cent. of my cases of jaundice, and find that it occurs with almost the same frequency in cancer of the pancreas and in janndice due to gall stones-8 per cent. and 9 per cent. respectively. I found sugar in the urine in 1 out of 8 cases of growth of the common duct. The quantity cannot be relied upon as an indication of the nature of the disease, for in cancer it has varied trom traces to about 2 per cent., and in gallstone cases from traces to 6 or 7 per cent., the gall-stone cases with high reccentages being all of old-standing. A small quantity of sugar is no bar to operation if proper precautions are taken and other indications suggest that it is advisable; in fact, it is not infrequently found that if the glycosuria is dependent upon an inflammatory lesion of the pancreas the sugar diminishes or disappears after operation, and with suitable medical and dietetio treatment the patient may live for many years. I have two patients who were operated on-one 12 and the other 14 years of ago-who are now in comparatively good health, except for the presence of sugar in their urine. Pancreatic diabetes due to cirrhosis of the gland, secondary to gall stones or duodenal ulcer, is, in my experience, not a very serious disease if the cause of the condition is recognized and means are taken to prevent the progress of the inflammatory changes at a sufficiently early stage. The improvement which follows operation in cases where there is obstruction of the ducts, is to be attributed to the relief of the tension within the ducts and to the subsidence of the associated catarrhal inflammation. The cirrhotic changes are permanent, and it depends upon the extent of these as to whether the glycosuria persists or not. But it must not be too readily assumed that, because the sugar disappears or is very much diminished after an operation the glycosuria has been cured. The rest in bed and comparative starvation are sometimes the chief factors in bringing this about, and when the patient returns to a more active life and a iuller diet the sugar reappears or increases. $\mathrm{He}$ is, however, in a better position to undergo a course of dietetic and medical trestment with hope of success, and if this is properly carried out, bearing in mind the cause of the condition, a further improvement will probably take place. Operative treatment is inadvisable when there is a large amount of sugar or an excess of ammonia nitrogen in the urine with acidosis, which is not readily controlled. The chief dangers, in addition to the risk of coma, which is considerable when there are over 2 grams of ammonia nitrogen in the urine in twentyfour hours, are sepsis and failure of the wound to heal. The former can be guarded against, but the latter hes proved in several cases with which I am acquainted a serious complication.

It is necessary to exclude the presence of sugar before proceeding to carry out the "pancreatic" reaction, as the dextrosazone crystels obscure the result. If sugar is found in the urine it may be removed in the way I described in an article on pancreatic diabetes in $1908,{ }^{1}$ but this method is not altogether satisfactory, especially in inexperienced hands, and whon the rationale of the process is not appre. ciated. I am at present engaged in perfecting a method of carrying out the "pancreatio" reaction with diabetic urines which is more rapid and appears to give more reliable results.

A positive pancreatic reaction in the urine points to there being some active degenerative changes of an inflammatory character in the pancreas at the time the urine was passed. It must be noted that no reaction is obtained in quiescent cirrhosis of the gland the result of past inflammation. I have obtained a positive reaction in 64 per cent. of the cases of chronic jaundice that I have examined, suggesting that in that proportion the condition was associated with, or was due to, an inflammatory lesion of the pancreas. In 30 per cent, that is to say in nearly half of these, the jaundice was dependent upon the presence of gall stones in the common bile duct. Common duct cholelithiasis does not necessarily give rise to chronic pancreatitis, for the gall stones may be in the supraduodenal or retroduodenal portions of the duct, where they do not come into relation with the pancreas, and it in the pancreatic portion this may ran behind the head of the pancreas instead of through it. Even when the intra. parietal portion of the duct is reached a small stone may pass without causing obstruction of the pancreatic duct if, as occasionally happens, the bile and pancreatic ducts enter the duodenum by separate openings instead of in the classical manner. Of my cases of common duct cholelithiasis 69.6 per cent. gave a positive pancreatic reaction, and 30.4 per cent. a negative result, which corresponds fairly closely with the 62 per cent. and 38 per cent. in which, sccording to Helly, the common bile duct lies imbedded in, and runs in a groove on the posterior surface of, the pancreas. If those in which no jaundice was present be excluded, 71 per cent. gave a positive reaction, and 29 per cent. a negative result. The slightly higher proportion giving a positive reaction than might theoretically be expected, especially in the joundiced cases, is probably to be explained by a simultaneous infection of the bile and pancreatic ducts in the way I have explained. An asso. ciated cholangitis and pancreatitis is also the probable explanation of the positive pancreatic reaction obtained with 9 per cent. of the cases in which gall stones were found only in the gall bladder at operation.

With primary malignant disease of the pancreas the pancreatic reaction is usually negative, but in about 30 per cent. of cases it is positive, owing probably to the inflammatory changes set up in the gland tissue by the blocking of the pancreatic duct and the irritation of the rapidly extending growth.

Next to gall stones and malignant disesse of the pancreas the most common diagnosis in chronic icterus is "catarrhal jaundice." It is usually, if not always, associated with gastro-intestinal disorders, and, owing to the close relation of the bile and pancreatic ducts to each other and to the duodenum, it is likely that there will be some pancreatitis in cases of this description. In the early stages the pancreatitis will be of the catarrhal type, resulting from obstruction of the ampulla of Vater and infection of the duct of Wirsung from the duodennm; but, if the intestinal catarrh remains unrelieved, and the accessory pancreatic duct is patent, the infection may be 
accentuated in the way I have previously mentioned, and marked swelling of the head of the pancreas follow. Should the case be one where the common bile duct passes through the head of the pancreas, the inflamed glend tissue will compress the bile duct, intensifying the jaundice, and, as Mayo Robson has suggested, causing it to persist much longer than is usual, so that a suspicion of gall stones, or even of malignant disease of the pancreas or common duot, may arise. The presence of a positive pancreatic reaction in 80 per cent. of the cases of catarrhal jaundice that I have examined shows how frequently the condition is associated with active degenerative changes in the pancreas. Fifteen of $\mathrm{my}$ cases in which the analysis of the urine had pointed to the presence of pancreatitis have been operated on, because of the persistence of the jaundice, and an enlarged and hard pancreas was found in them all.

Growths of the common bile duct may or may not give rise to pancreatitis and a pancreatic reaction in the urine according to their situation and extent. I have had three cases of malignant disease of the ampulla of Vater and each gave a positive reaction, the growth acting like an impacted gall stone in that situation. Cancer of the upper portion of the duct or of the gall bladder may not at first cause pancreatitis, but as the growth extends down the duct it will set up inflammatory changes in the gland. Two of my cases of cancer of the common duct gave a positive pancreatic reaction and six a negative result. The jaundice in twelve of my cases was due to metastatic deposits of growth from primary malignant disease in various situations, and eleven of these gave a positive pancreatic reaction, pointing to the pancreas being invaded by the growth.

It will be seen that, while the pancreatic reaction in the urine is of value as showing the presence or absence of pancreatitis in cases of persistent jaundice, it does not by itself differentiate between the causes, which may be gall stones in the common duct, an ascending infection from the duodenum, malignant disease of the pancreas, or more rarely a growth of the common duct. In addition, the possibility of an inflammatory condition of the gland from disease of the heart, lungs or blood vessels, tubercle, 8yphilis, etc., with jaundice from other causes, has to be borne in mind. To make a satisfactory diagnosis, the whole of the results to be obtained by a thorough quali tative and quantitative analysis of the urine and faeces must b6 taken into account, and these again be checked by a considerstion of the clinical signs and symptoms.

An analysis of the faeces is of importance in all cases of obstructive jaundice, and is particularly valuable where there is a suspicion of malignant disease. The percentage of unabsorbed fats, the relation between the saponified and unsaponified fats, the presence of stercobilin and occult blood, the results of the pancreatic insufficiency test, and the proportion of inorganic ash must be determined and carefully considered, for from them one can determine with a considerable degree of accaracy the extent of the pancreatic mischief, also whether the biliary obstruction is complete or not, and then infer whether one is dealing with a case of growth or simple obstruction.

The effects of the exclusion of bile trom the intestine are shown in the faeces mainly by (1) defective absorption of fat; (2) an increase in intestinal putrefaction; and (3) loss of the faecal colouring matter.

Fats.

In the stomach fats undergo little or no change, but when they reach the small intestine they are attacked and broken down by the sterpsin of the pancreatic juice into fatty acids and glycerine. A variable proportion of the former unites with the alkalis o the intestinal secretions to form soaps. The soaps thus formed, the uncom. bined fatty acids, and the glycerine are absorbed by the epithelial cells of the intestinal wall, and are there synthesized to again form neutral fats. The presence of bile favours the action of the pancreatic secretion by helping in the emalsification of the fat, through its alkalinity, and by directly reinforcing the activity of the tryptic ferment. It also makes the free fatty acids, which are insoluble in water, soluble, and increases the solubility of the alkaline soaps. If, therefore, the bile is excluded from the intestine the total amount of fat in the faeces will be increased and the greater part should consist of combined fatty acids or soaps. Such, in fact, is usually found to be the case in simple gall-stone obstruction and malignant disease of the common bile duct, or gall bladder, when the fat-splitting functions of the pancreas are being normally carried out. If, however, the digestive powers of the pancreas are interfered with, from obstruotion of the duct of Wirsung by a stone, malignant disease of the head of the glend, or advanced cirrhosis, the cleavage of fats is also defective and they appear in the faeces mainly in the form of neutral fats and free fatty acids. In a typical case it is consequently possible to say whether there is simple biliary obstruction or serions interference with the digestive functions of the pancreas from one or other of the causes I have mentioned, by estimating the fats in the faeces and determining the proportions of saponified and ansaponified fats. The absence of digestive ferments does not, however, cause an entire cessation of the fat-splitting process, for, even in health, a certain amount of fat is broken down into fatty acids and glycerine through the activity of organisms of the colon group in the lower part of the small and upper part of the large intestine. If the activity and number of these bacteria is increased, as appears to be the case in intestinal catarrhs, they mey bring about a considerable amount of fat digestion, but, since the fatty acids formed in this way are not absorbed during their passage through the colon and sigmoid but combine with alkalis, they appear in the faeces as sosps, chiefly of calcium and magnesium. Hence in catarrhal conditions of the intestine and also in catarrhal janndice, it is usually found that the combined fatty acids are in excess and that the inorganic ash is also increased.

The greatest increase in the amount of total fat is seen in cancer of the pancreas, where $I$ have tound as much as 93 per cent. of the dry weight of the faeces to consist of fat. In gall-stone cases where there is jaundice and pancreatitis the total-fat in my cases has averaged 56 per cent., and in 60 per cent. of them the unsaponified have been in excess of the seponified fats. Simple jaundice due to gall stones, where there has been no involvement of the pancreas, has given about the same reading of total fat in many instances, but in 75 per cent. the saponified have exceeded the unsaponified fats. Growths of the common bile duct, or gall bladder involving the common duct, produce much the same effects as gall stones, the saponified exceeding the unsaponified fats, except when the growth is situated at the ampulla of Vater or is invading the pancreas, when the unsaponified fats are in excess.

Intestinal Putrefaction.

The antiseptic action of bile has been much overrated. Its influence on putrefactive bacteria is very slight, and is quickly lost. Indirectly, however, the bile in the intestine has an important function in keeping the putrefactive processes within bounds by stimulating peristalsis, and so preventing stagnation of the intestinal contents. It also acts indirectly on putrefactive processes by aiding fat absorption, and so allowing more complete digestion of the proteid elements of the food. Absence of bile from the intestine, therefore, tends to favour constipation and irregular action of the bowels. The effects of the consequent excessive putrefaction are shown by the offensive smell and markedly alkaline resction of the stools, and are seen in the urine in the shape of an increase in the ethereal sulphates, an excess of phenol and aromatic oxyacids, and sometimes by a well-marked reaction for indican. These effects are more marked when there is an associated intestinal catarrh, and are more commonly seen in catarrhal jaundice than in jaundice due to gall-stone obstruction. The presence of pancreatic disease modifes the condition of the stools. If the functions of the pancreas are seriously interfered with, the motions become more bulky, often very strikingly so, are more frequent, and have an acid reaction; the odour, too, is somewhat different, resembling that of rancid bacon.

\section{Colour.}

Complete diversion of the bile from the intestine gives to the faeces a light grey or slaty tint, producing the so-called "clay-coloured" stools. It should be remembered, however, that although the presence of the normal brown colour of the faeces may be taken as satisfactory evidence that bile is freely passing into the intestine, the existence of "clay.coloured" motions is not of itself a conclusive 
sign that there is complete biliary obstruction. This is a most important point to determine with certainty, for while complete obstruction is the exception in gall-stone cases, it is the rule when the obstruction is due to malignant disease of the head of the pancreas. Chemical examination of the faeces in the cases $I$ have investigated has shown that in over 60 per cent. with cancer of the pancreas no stercobilin could be found, and in 34 per cent. only traces could be discovered, whereas traces at least of stercobilin were found in every instance where the obstruction was due to gall stones, and in 82 per cont. a well-marked reaction was obtained, in spite of the fact that in many of them the faeces were quite white to the naked eye. Traces of stercobilin are usually found in malignant disease of the common bile duct and cancer of the gall bladder invading the common duct, for in these the growth is usually of a soft character and allows a certain amount of bile to percolate through, unlike the scirrhous growth of the head of the pahcreas which compresses the duct tightly from without. The white appearance of the faeces is usually most striking when the obstruction of the bile flow is associatod with well-marked pancreatic disease. The peculiar glistening, silvery character of the motions in these cases appears to be due partly to the presence of fatty acid orystals, which act upon light like snow and other finely crystalline bodies, and partly to the action of anaërobic bacteria, which reduce any bile pigment that may be present to a colourless form.

In catarrhal jaundice the unabsorbed fat is intimately mixed with the other constituents of the faeces and the exudates from the intestinal walls, so that the motions are of a light-grey colour and have a peculiar salve-like consistency. They give a well.marked reaction for stercobilin on chemical analysis.

The Pancreatic Insufficiency Tests.

The pancreatic insufficiency tests of Gross and Heiberd give useful indications as to the functional activity of the pancreas, but they only show very gross changes in the digestive powers of the gland, and so are of limited use when taken alone. A negative pancreatic insufficiency test-that is to say, one in which there is normal casein digestion-shows that there is no serious interference with the digestive functions of the pancreas, and in jaundice cases is against a diagnosis of malignant disease of the pancreas. A well-marked positive result points to malignant disease, advanced cirrhosis of the gland, or blocking of the duct of Wirsung by a gall stone impacted in the ampulla of Vater. In cirrhosis of the pancreas and blocking of the duct by gall stones one almost always finds, however, that there is some casein digestion, so that the more incomplete the digestion the more likely is it that the jaundice is due to obstruction of the common bile duct by malignant disesse of the head of the pancreas.

\section{Occult Blood}

The constant presence of occalt blood in the freces is suggestive of malignant disease somewhere in the course of the gastro-intestinal tract. Its intermittent presence points to simple ulceration. In cases of cancer of the pancreas, common bile duct, or gall bladder, occult blood occurs in the faeces with great constancy, so that a positive reaction in a case of deep jaundice, especially if it is found on four or five consecutive days, points to the jandice being due to a growth in one or other of these situations. A positive result may also be due to malignant disease of the stomach or intestine with glands in the portal fissure, or more rarely to advanced simple chronic pancreatitis, bat a consideration of the results obtained by other methods of examination will usuall p decide to which of these it is to be attributed.

\section{Inorganic Ash.}

Normally the inorganic ash of the raeces constitutes from 10 to 15 per cent. of the dry weight, bat when from any cause there is a catarrh of the intestinal walls this percentage is considerably increased. One, theretore usually finds that there is an excess of inorganic ash in catarrhal janndice and in cases where the jaundice is secondary to growths of the intestinal canal, particularly

the colon.
By a timely and correct diagnosis, such as is possible from a careful consideration of the clinical history and symptoms, and the information to be obtained by a thorough analysis of the urine and faeces, the complications and sequelae likely to arise in cases of chronic jaundice, such as serious disease of the pancreas, cholangitis, cirrhosis of the liver, etc., may be avoided. Delay is always dangerous, and it is useless to await developments until the case is only of interest to a pathological anatomist. The cause of the janndice mast be determined at the earliest possible moment and the treatment be based upon the conclusion arrived at. A diagnosis of gall stones obstructing the bile duct, made on the clinical data and confirmed by an examination of the excreta, calls for immediate operation, as the only likely spontaneous cure is Nature's crude and dangerous remedy of making a fistula by which the stone may escape into the duodenum, or elsewhere. Catarrhal jaundice very frequently clears up spontaneously, but if after six weeks' rest in bed, with dieting and medical treatment, the jaundice persists and the urinary "pancreatic" reaction is positive, it is advisable to invoke the aid of the surgeon to perform a short. circuiting operation to avoid further damage to the pancreas. In the last stages of malignant disease operation is only likely to shorten the brief span of life still left to the patient, but in the earlier stages much may be done to make existence more bearable by a cholecystotomy or cholecystenterostomy to relieve the intolerable itching of which complaint is chiefly made. At the same time it is well to bear in mind that many cases diagnosed clinically as inoperable carcinoma of the pancreas have been shown on chemical examination of the urine and faeces, and by their after-history, to be suffering from chronic inflammation of the gland capable of retrogression.

1 Surgery, Gynaecology, and Obstetrics, January, 1908, p. 22.

\section{OBSERVATIONS ON THE URINES OF MARATHON RUNNERS.}

By GRAHAM CHAMBERS, B.A., M.B.,

ASSOCIATE PROFESSOR OF OLINICAL MEDICINE, UNIVERSITY OF TORONTO: PHYSICIAN, TORONTO GENERAL HOSPITAL.

SrNce von Leube in 1877 reported his observations on the urines of soldiers a number of reports bearing on the relation of albuminuria to physical exertion have appeared, but still the literature is not as plentiful as one might desire. It occurred to me, therefore, that some observ8. tions made on urines of Marathon runners might prove instructive and of value to those interested in the subject.

The subjects of the investigation were eight athletes who competed in a Marathon race at Toronto on May 24th, 1910. The race began at 8.30 p.m., and three hours before the contestants ate fairly heary meals of beef, bread, butter, etc. The urines were obtained immediately before and again immediately after the race, and analysed, tests being made for " nucleo-albumen " (Mörner body), albumen, globulin, sugar, and indican, and the sediment examined' microscopically.

The resalts showed several interesting features. The urine of one contestant, who, on account of abdominal pain. and vomiting, was forced to quit in the seventh mile, con. tained a great excess of indican both before and after the race. I may mention that this runner's previous records had been the best of all the contestants, and in this race he was looked upon as a likely winner.

This observation shows the importance of keeping the digestion of an athlete in a healthy condition, a point which I think is recognized by trainers.

Another interesting feature was the presence of hyalin. and faintly granular casts in a urine voided after the race, although "nucleo-albumen," albumen and globalin were absent.

One might also make special mention of the presence of calcium oxalate crystals in the sediments of five of the eight specimens collected before the race. After the contest the urines of the same runners contained the same crystals in their sediments, but they were much less plentiful in each specimen. Indeed, in three of the five, they were found only atter examining several slides. 